This item was submitted to Loughborough's Research Repository by the author.

Items in Figshare are protected by copyright, with all rights reserved, unless otherwise indicated.

\title{
On relationship between time-domain and frequency-domain disturbance observers and its applications
}

PLEASE CITE THE PUBLISHED VERSION

http://dx.doi.org/10.1115/1.4033631

\section{PUBLISHER}

(c) American Society of Mechanical Engineers (ASME)

VERSION

AM (Accepted Manuscript)

\section{PUBLISHER STATEMENT}

This work is made available according to the conditions of the Creative Commons Attribution-NonCommercialNoDerivatives 4.0 International (CC BY-NC-ND 4.0) licence. Full details of this licence are available at: https://creativecommons.org/licenses/by-nc-nd/4.0/

\section{LICENCE}

CC BY-NC-ND 4.0

\section{REPOSITORY RECORD}

Su, Jinya, Wen-Hua Chen, and Jun Yang. 2016. "On Relationship Between Time-domain and Frequencydomain Disturbance Observers and Its Applications”. figshare. https://hdl.handle.net/2134/21201. 


\title{
On Relationship between Time-Domain and Frequency-Domain Disturbance Observers and Its Applications
}

\author{
Jinya Su* \\ Department of Aeronautical \\ and Automotive Engineering, \\ Loughborough University, \\ Loughborough, LE11 3TU, U.K. \\ Email: J.Su2@lboro.ac.uk
}

\author{
Wen-Hua Chen \\ Department of Aeronautical \\ and Automotive Engineering, \\ Loughborough University, \\ Loughborough, LE11 3TU, U.K. \\ Email:W.Chen@lboro.ac.uk
}

\author{
Jun Yang \\ School of Automation, \\ Southeast University, \\ Nanjing 210096, China. \\ Email: j.yang84@seu.edu.cn
}

\begin{abstract}
This paper provides a generic analysis of the relationship between timelfrequency-domain $D O B$ design methodology. It is discovered that the traditional frequency-domain DOBs using a low pass filter with unity gain can only handle disturbances satisfying matching condition, while the traditional time-domain DOBs always generate an observer with a high order. A Functional Disturbance OBserver (FDOB) is proposed to improve the existing results together with its design guideline, frequency analysis and existence condition. Compared with the existing frequency-domain DOBs, the proposed FDOB can handle more classes of disturbances, while compared with the existing time-domain DOBs the proposed $F D O B$ can generate an observer with a lower order. Numerical examples are presented to illustrate the main findings of this paper including a rotary mechanical system of nonminimum phase.
\end{abstract}

Keywords: Functional Disturbance OBserver (FDOB); reduced order; non-minimum phase; time/frequency domain design; ADRC.

\section{Introduction}

In industrial control systems, disturbances and uncertainties widely exist, which bring adverse effects on control performance or even affect system stability (see, [1-5]). A large number of disturbance attenuation and robust control methods have been proposed, among which Disturbance Observer Based Control (DOBC) has received much attention $[4,6,7]$ due to its promising features such as the preservation of the nominal performance and the "separation principle" for the ease of control design [4]. In DOBC de- sign, there are two control loops designed separately, i.e., the outer loop baseline controller addressing nominal performance specifications and stability, and the inner loop Disturbance OBserver (DOB) rejecting disturbances and suppressing uncertainties $[2,4,7]$. In the absence of disturbances and uncertainties, the inner loop DOB is not activated and consequently the DOBC reduces to the baseline controller $[2,8]$. As a result, unlike the worst-case robust control approaches where nominal control performance is sacrificed to achieve better robustness, the nominal performance can be preserved in DOBC [7].

The key element in DOBC is the design of DOB to estimate disturbances and uncertainties [2,4]. There are two distinct approaches for linear systems including time-domain DOBs [9-11] and frequency-domain DOBs $[1,12,13]$. The time-domain DOB firstly appeared in the late 1960s when Johnson [9] first developed the Disturbance Accommodating Control by proposing Unknown Input Disturbance Observer (UIDO). Recently, from different prospects, Han [10] developed Active Disturbance Rejection Control (ADRC) through the technique of Extended State Observer (ESO). The key idea is to firstly augment the plant with disturbances/uncertainties and then construct an observer estimating the augmented states including the disturbances. Frequency-domain DOB was originally proposed by [12]. The original idea is to obtain disturbance estimate by filtering the differences between control input and calculated input using the inverse model of nominal plant. This approach has been further developed to achieve robust stability in [13]. Recently, much attention has been paid to embedding internal model of disturbances (similar to internal model principle) in DOBs such that a larger class of disturbances (e.g., sinusoidal) can be handled $[14,15]$. 
These two types of DOBs were developed from different prospects with different design principles and tools. So far, little literature is available to investigate the relationship between them except [1]. The authors of [1] pioneered the study of the relationship between them by analysing their structure and transfer functions. It was concluded that the frequency-domain DOB is a generalization of time-domain $\mathrm{DOB}$, since there is less design freedom in time-domain DOB in choosing the order and relative degree of the transfer function from control input to disturbance estimate, and the time-domain DOB has a higher order in comparison with frequency-domain DOB. However, it shall be highlighted that both the system model and disturbance model are supposed to be in an observable canonical forms in time-domain DOB in [1]. Therefore it may not be easy to see how the disturbance model is incorporated in the corresponding transfer functions of the time-domain DOB. Consequently, as pointed out in [1], it becomes hard to select the equivalent low-pass filter in frequency-domain DOB to handle generic disturbances for non-minimum phase systems.

This paper first presents a generic analysis of the relationship between time-domain and frequency-domain DOB design methods. The system and disturbance models of the time-domain DOB in this paper are in a generic form. As a result, it is explicitly pointed out how the system model and disturbance model are incorporated in the equivalent transfer function realisation of the DOB designed in state-space approach. It is also discovered that the traditional frequencydomain DOB employing a low pass filter with unity gain is only able to handle a specific class of disturbances satisfying the matching condition $[4,16]$.

As pointed out in [1], compared with frequency-domain DOB, the existing time-domain DOB generates an observer of a higher order. Consequently, this paper then proposed a new type of time-domain DOB (termed Functional Disturbance OBserver (FDOB)). The FDOB is inspired by pioneering work on reduced-order DOB [17-19], where the DOB is designed by estimating disturbances and state function instead of all the states. However, our design methodology is quite different from them. The proposed FDOB reduces the observer order by combining the idea of augmenting system states with disturbances and the functional observer theory in [20]. Detailed discussion on FDOB is given including observer structure, transfer function implementation, and existence condition. Compared with frequency-domain DOB, the proposed FDOB can directly handle more classes of disturbances (matched or mismatched, high-order, harmonic disturbances, and their combinations), while compared with the traditional time-domain $D O B$, the proposed FDOB can generate an observer with a lower order.

The rest of the paper is outlined as follows: In Section 2, preliminaries on linear DOBs are given. In Section. 3, the relationship between time-domain DOB (i.e., UIDO in DAC and ESO in ADRC) and frequency-domain DOB is discussed, on which basis the motivations of the paper are presented. In Section 4, FDOB is proposed together with its transfer function realisation, existence condition and design procedure. In Section. 5, the proposed FDOB is imple-

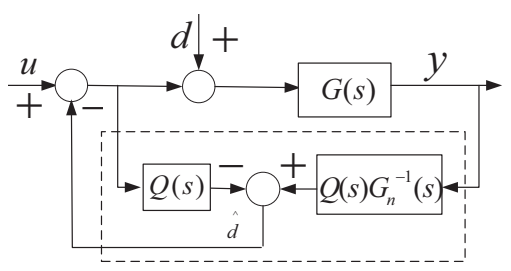

Fig. 1. The diagram of classic $Q$-filter based DOB in [12].

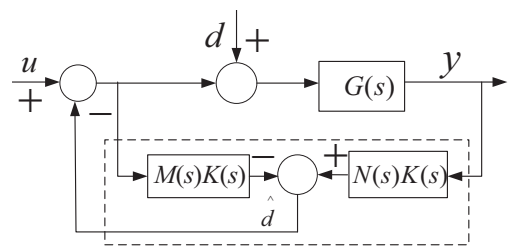

Fig. 2. The diagram of a generic DOB structure.

mented in frequency-domain such that the frequency-domain DOB can accommodate generic disturbances. In Section 6, several examples are given to illustrate the findings of the paper. Section 7 concludes the paper.

\section{Preliminaries}

In this section, preliminaries on frequency-domain DOBs in $[1,12]$ and time-domain DOBs in $[9,10]$ are briefly reviewed.

\subsection{Frequency-domain DOBs}

The frequency-domain DOBs were originally proposed in [12]. Suppose the transfer function is $G(s)$, the basic idea is to obtain disturbance estimate by filtering the differences between control input and calculated input using the inverse model of nominal plant $G_{n}(s)$. Its basic diagram $[1,12]$ is given in Fig. 1, where $Q(s)$ is designed as a low-pass filter with unity gain and the relative degree of $Q(s)$ is no less than that of the nominal plant $G_{n}(s)$ such that $Q(s) G_{n}^{-1}(s)$ is implementable. The estimate of lumped disturbance in DOB Fig. 1 is given by

$$
\begin{gathered}
\hat{d}(s)=G_{u \hat{d}}(s) u(s)+G_{y \hat{d}}(s) y(s), \\
G_{u \hat{d}}(s)=-Q(s), G_{y \hat{d}}(s)=G_{n}^{-1}(s) Q(s) .
\end{gathered}
$$

However, the original structure in [12] can not effectively handle systems of non-minimum phase since the direct inverse of nominal plant $G_{n}(s)$ brings unstable poles in $Q(s) G_{n}^{-1}(s)$. An improved version of DOB is given in [1], which can be equivalently represented in Fig. 2, where M(s) and $\mathrm{N}(\mathrm{s})$ take the following form

$$
M(s)=M_{n}(s) / L_{1}(s), N(s)=N_{n}(s) / L_{1}(s),
$$

where $G_{n}(s)=M_{n}(s) / N_{n}(s), L_{1}(s)$ is a stable polynomial, $K(s)=K_{n}(s) / L_{2}(s)$ is designed as a low-pass filter such that $M(s) K(s)$ is a low-pass filter with unity gain.

\subsection{Time-domain DOBs}

In this subsection, two typical types of time-domain disturbance estimation approaches are presented including UIDO in DAC [9] and ESO in ADRC [10]. 


\subsubsection{UIDO in DAC}

The philosophy of time-domain DOB proposed in [9] is that disturbance estimate can be obtained by simultaneously estimating the augmented states consisting of state dynamics and disturbance dynamics. The mathematical interpretation is as follows. Consider a Single-Input-Single-Output (SISO) uncertain linear system

$$
\left\{\begin{array}{l}
\dot{x}=A x+B u+D d \\
y=C x
\end{array},\right.
$$

where $x \in R^{n}, u \in R, d \in R$ and $y \in R$ are system states, control input, disturbance and measurement, respectively. $A, B, C, D$ are the corresponding system matrices, which can be considered as a state-space realization of the nominal plant $G_{n}(s)$ in Fig. 1. If disturbance $d$ does not enter the system as the same channel of input $u$ then $d$ becomes a mismatched disturbance $[8,21]$.

The disturbance is supposed to be generated by a linear exogenous system [22]

$$
\dot{\omega}=S \omega, d=H \omega,
$$

where $\omega \in R^{q}$, the pair $(S, H)$ is known and observable, and the initial states are unknown. System (3) can accommodate the vast majority of disturbances encountered in practical engineering, such as constants, ramps, polynomials in time, exponentials, sinusoids and their combinations.

To facilitate the discussion, a definition is given to categorize different disturbance models.

Definition: If the matrix $S$ in (3) satisfies $\operatorname{det}(S)=0$, then the disturbance is defined as Type I disturbance; otherwise, the disturbance is defined as Type II disturbance for $\operatorname{det}(S) \neq 0$.

Remark 1: It should be noted that the widely investigated high-order disturbance is a special case of Type I disturbance, e.g., constant disturbance when $S=0$ and $H=1$ in $[9,10]$ and $r$-th polynomial disturbance when $S=\left[\begin{array}{cc}O_{(r-1) \times 1} & I_{r-1} \\ 0 & O_{1 \times(r-1)}\end{array}\right]$ and $H=\left[1 O_{1 \times(r-1)}\right]$, which is the case investigated by high order disturbance observer in $[1,23]$, generalized ESO in [24], Generalized Proportional Integral (GPI) observer in [25]. On the other side, harmonic disturbance represented by $S=\left[\begin{array}{cc}0 & \lambda \\ -\lambda & 0\end{array}\right]$ with $\lambda \neq 0$ and $H=\left[\begin{array}{ll}1 & 0\end{array}\right]$ (e.g. [22]) is a special case of Type II disturbance.

Combining system dynamics (2) and disturbance dynamics (3), a composite system can be obtained

$$
\left\{\begin{array}{l}
\dot{\tilde{x}}=\tilde{A} \tilde{x}+\tilde{B} u \\
y=\tilde{C} \tilde{x}
\end{array}\right.
$$

where $\tilde{x}=\left[x^{T}, \omega^{T}\right]^{T}$, the system matrices are given by

$$
\tilde{A}=\left[\begin{array}{cc}
A & D H \\
O_{q \times n} & S
\end{array}\right], \tilde{B}=\left[\begin{array}{c}
B \\
O_{q \times 1}
\end{array}\right], \tilde{C}=\left[\begin{array}{ll}
C & O_{1 \times q}
\end{array}\right] .
$$

Under the detectability condition of the matrix pair $(\tilde{A}, \tilde{C})$, an observer for (4) can be designed as

$$
\left\{\begin{array}{l}
\dot{\hat{\tilde{x}}}=\tilde{A} \hat{\tilde{x}}+\tilde{B} u+K(y-\tilde{C} \hat{\tilde{x}}) \\
\hat{\omega}=\tilde{C} \hat{\tilde{x}}, \hat{d}=H \hat{\omega}
\end{array},\right.
$$

where $K$ is the observer gain matrix to be designed (e.g. pole assignment of the matrix pair $(\tilde{A}, \tilde{C}))$ and $\tilde{C}=\left[O_{n}, I\right]$.

\subsubsection{ESO in ADRC}

Consider a SISO (possibly non-linear) system with disturbance, depicted by

$$
y^{(n)}(t)=f\left(y(t), \dot{y}(t), \cdots, y^{(n-1)}(t), d(t), t\right)+b u(t),
$$

where $y^{(l)}$ denotes $l$ th derivative of the output $y$, and $u$ and $d$ denote input and disturbance, respectively. To simplify the notation, the time variable will be dropped if no confusion is caused. Letting $x_{1}=y, x_{2}=\dot{y}, \cdots, x_{n}=y^{(n-1)}$, one has

$$
\left\{\begin{array}{l}
\dot{x}_{i}=x_{i+1}, i=1, \cdots, n-1 \\
\dot{x}_{n}=f\left(x_{1}, x_{2}, \cdots, x_{n}, d\right)+b u
\end{array} .\right.
$$

Choose a new state $x_{n+1}=f\left(x_{1}, x_{2}, \cdots, x_{n}, d\right)$, then its derivative is given by

$$
\dot{x}_{n+1}=h(t) \text {, with } h(t)=\dot{f}\left(x_{1}, x_{2}, \cdots, x_{n}, d\right) .
$$

ESO is designed to estimate all the states and lumped disturbance term $f$, given by [10]

$$
\left\{\begin{array}{l}
\dot{\hat{x}}_{i}=\hat{x}_{i+1}+\beta_{i}\left(y-\hat{x}_{1}\right), i=1, \cdots, n, \\
\dot{\hat{x}}_{n+1}=\beta_{n+1}\left(y-\hat{x}_{1}\right) .
\end{array}\right.
$$

Define the estimation error as $e_{i}=x_{i}-\hat{x}_{i}$, one obtains the error dynamics of ESO

$$
\left\{\begin{array}{l}
\dot{e}_{i}=e_{i+1}-\beta_{i} e_{1}, i=1, \cdots, n, \\
\dot{e}_{n+1}=-\beta_{n+1} e_{1}-h(t) .
\end{array}\right.
$$

The gains shall be chosen such that the polynomial

$$
s^{n+1}+\beta_{1} s^{n}+\cdots+\beta_{n} s+\beta_{n+1}=0,
$$

is Hurwitz. In ESO design, both the model dynamics and uncertainties are estimated, where only the system relative degree is required. So the significant feature of ESO is that it requires a minimum information about a dynamic system (see [26] for various extensions).

\section{Relationship between time/frequency-domain 3.1 UIDO VS frequency-domain DOBs}

First the relationship between UIDO and frequencydomain DOB is discussed. To this end, frequency-domain analysis is performed on time-domain DOB (5) to derive a set of transfer functions. Compared with [1], a generic system model (2) and disturbance model (3) are considered in this paper, which explicitly shows how they are incorporated in the transfer functions.

From (5), we can obtain the transfer functions from control input $u(s)$ and measurement $y(s)$ to disturbance estimate $\hat{d}(s)$ using Laplace transformation, given by

$$
\begin{gathered}
\hat{d}(s)=-G_{u \hat{d}}(s) u(s)+G_{y \hat{d}}(s) y(s), \\
G_{u \hat{d}}(s)=-H \tilde{C}[s I-(\tilde{A}-K \tilde{C})]^{-1} \tilde{B}, \\
G_{y \hat{d}}(s)=H \tilde{C}[s I-(\tilde{A}-K \tilde{C})]^{-1} K, K=\left[K_{1}^{T} K_{2}^{T}\right]^{T} .
\end{gathered}
$$

The transfer function realisation of DOB (5) is given in Fig. 3 . To explicitly find out the relationship between transfer functions $G_{u \hat{d}}(s), G_{y \hat{d}}(s)$ and the system/disturbance models (2), (3), theoretical analysis is performed on (8) and (9), and the results are summarized in Theorem 1. 


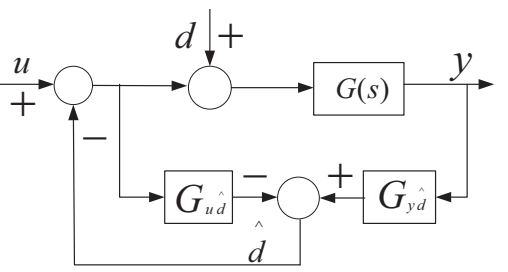

Fig. 3. Frequency-domain interpretation of time-domain DOBs.

Theorem 1. For linear system (2) with disturbance model (3), if the time-domain DOB is designed as (5), then its transfer function realisation (7) is given by

$$
\begin{aligned}
G_{u \hat{d}}(s) & =\frac{\operatorname{Hadj}(s I-S) K_{2} M_{n}(s)}{\operatorname{det}(s I-(\tilde{A}-K \tilde{C}))}, \\
G_{y \hat{d}}(s) & =\frac{\operatorname{Hadj}(s I-S) K_{2} N_{n}(s)}{\operatorname{det}(s I-(\tilde{A}-K \tilde{C}))},
\end{aligned}
$$

where $M_{n}(s)$ and $N_{n}(s)$ are the numerator and denominator of the nominal plant $G_{n}(s)$.

The proof is given in Appendix A.

Remark 2: Different from [1], it can be explicitly seen from (10) and (11) how system model (i.e., the system matrices $(A, B, D, C))$ and disturbance model (i.e., the pair $(S, H))$ of the DOB (5) are incorporated into the transfer functions.

\subsubsection{Equivalence between UIDO and frequency- domain DOBs}

Comparison results between UIDO (5) and frequencydomain DOB in Fig. 2 are summarized as follows.

i. The denominator of (10) can be factored into $\operatorname{det}(s I-$ $(\tilde{A}-K \tilde{C}))=L_{1}(s) L_{2}(s)$. Consequently, (10) and (11) can be reformulated into the same format of $M(s) K(s)$ and $N(s) K(s)$ in Fig. 2 by treating $K_{n}(s):=\operatorname{Hadj}(s I-$ S) $K_{2}$

ii. One can obtain from Fig. 2 and 3 that they share a same structure in transfer function form by treating $M(s) K(s)$ as $G_{u \hat{d}}$ and $N(s) K(s)$ as $G_{y \hat{d}}$;

iii. For Type I disturbance (see, Definition 1) under matching condition (i.e., $\mathrm{D}=\mathrm{B}$ ), one can prove that

$$
G_{u \hat{d}}(0)=1
$$

which means $G_{u \hat{d}}$ is a low-pass filter with unity gain. The proof is given in Appendix B.

iv. One can obtain the following identity

$$
\frac{1}{1-G_{u \hat{d}}}=\frac{\operatorname{det}(s I-(\tilde{A}-K \tilde{C}))}{\operatorname{det}\left(s I-A+K_{1} C\right) \operatorname{det}(s I-S)} .
$$

The proof is given in Appendix C. We can see from (13) that $1 /\left(1-G_{u \hat{d}}\right)$ includes the disturbance model information $1 / \operatorname{det}(s I-S)$. The need of $1 /\left(1-G_{u \hat{d}}\right)$ including the disturbance model has been identified in [1] but it is hard to choose $Q(s)$ to implement it.

\subsubsection{Relationship with the results in [1]}

Our results significantly extend the celebrated work in [1]. First, the aforementioned Points i and ii reduce to the results in [1] when the system dynamics (2) and disturbance dynamics (3) take the special observable canonical structure, and the frequency-domain DOB in Fig. 2 reduces to the traditional one in Fig. 1. This can be obtained by selecting

$$
L_{1}(s)=M_{n}(s), K(s)=Q(s) .
$$

In Point iii, we prove that $G_{u \hat{d}}=1$ holds only for Type I disturbance satisfying matching condition. Specifically, the high order disturbance $1 / s^{n}$ discussed in [1] is a special case of matched Type I disturbance. Based on our results, the traditional frequency-domain DOBs using low pass filter $Q(s)$ with unity gain in Fig. 1 can only handle matched Type I disturbance and fail to handle mismatched disturbance or Type II disturbance such as harmonic disturbance.

It has been pointed out in [1] it is not trivial to select $G_{u \hat{d}}$ to handle generic disturbance for non-minimum phase system, but our Theorem 1 and Point iv explicitly point out how $G_{u \hat{d}}$ contains the unstable zeros of $M_{n}$ and $1 /\left(1-G_{u \hat{d}}\right)$ includes the disturbance model information $1 / \operatorname{det}(s I-S)$.

\subsection{ESO VS frequency-domain DOBs}

Second, it is of much interest to compare DOBC in Fig. 1 [12] with the ADRC in [10]. Their major difference results from different disturbance estimator, where DOBC utilizes DOB while ADRC employs Extended State Observer (ESO). We will elaborate the relationship/difference between them by comparing them using system (2).

Taking $y$ and $u$ as the inputs of ESO in (6), the ESO observer dynamics for system (2) are gives by

$$
\left[\begin{array}{l}
\dot{\hat{x}} \\
\dot{\hat{d}}
\end{array}\right]=\tilde{A}\left[\begin{array}{l}
\hat{x} \\
\hat{d}
\end{array}\right]+\tilde{B}_{u} u+\tilde{B}_{y} y \text {. }
$$

where the matrices are given by

$$
\tilde{A}=\left[\begin{array}{cc}
A & D \\
0 & 0
\end{array}\right], \tilde{B}_{u}=\left[\begin{array}{c}
B \\
0
\end{array}\right], \tilde{B}_{y}=L=\left[L_{x}^{T} L_{d}^{T}\right]^{T}, \tilde{C}=[\underbrace{0 \cdots 0}_{n} 1] \text {. }
$$

Taking disturbance estimate $\hat{d}$ as the output of ESO, the transfer function formulation of the ESO is

$$
\begin{gathered}
\hat{d}(s)=G_{u \hat{d}}(s) u(s)+G_{y \hat{d}}(s) y(s), \\
G_{u \hat{d}}(s)=\tilde{C}(s I-\tilde{A})^{-1} \tilde{B}_{u}, \\
G_{y \hat{d}}(s)=\tilde{C}(s I-\tilde{A})^{-1} \tilde{B}_{y} .
\end{gathered}
$$

Theorem 2. Consider a system described by (2) with $B=$ $D$, and the ESO is designed by (14), if let

$$
Q(s)=-\tilde{C}(s I-\tilde{A})^{-1} \tilde{B}_{u},
$$

then the following two facts hold: 1) $Q(s)$ is a stable lowpass filter with a unity $d c$ gain if the observer gains are appropriately designed; 2) (15) and (16) become $G_{u \hat{d}}(s)=$ $-Q(s)$ and $G_{y \hat{d}}(s)=G_{n}^{-1}(s) Q(s)$, which are the same as those in (1) of frequency-domain DOB.

The proof is given in Appendix D. Theorem 2 implies that the ESO (6) is equivalent to the frequency-domain DOB in Fig. 1 for system (2) when $Q(s)$ filter is chosen to have an order of $n+1$ where $n$ is the order of the system.

Remark 3: ADRC was developed as an alternative to the classic PID so it aims at requiring minimum model information. Therefore, it can be applied to a wide variety of systems without requiring too much modelling effort, but this 
very strength also brings its weakness: it does not exploit the modelling information even if it is available. DOBC provides a powerful approach to exploit modelling information. Rather than representing the plant as an integrator chain, it is able to use the available modelling information if it is available but treated as the mismatching between the nominal model and the physical system if it is not available.

\subsection{Motivations: gaps of time/frequency-domain DOBs}

Although the frequency-domain DOB is equivalent to the frequency-domain in terms of structure, there still exist some gaps summarized in the following sections.

\subsubsection{Observer order and minimum relative degree}

First, the observer order of time-domain DOB is higher than that of frequency-domain DOB for plants with stable zeros (see, pp. 546 of [1]). Second, the minimum relative degree of $G_{u \hat{d}}$ of time-domain DOB is higher than that of frequency-domain DOB. As pointed out in [1], the relative degree of equivalent $G_{u \hat{d}}$ is larger than or equal to the relative degree of the nominal plant, so the minimum relative degree of $G_{u \hat{d}}$ in frequency-domain DOB as given in Fig. 2 can be chosen to be equal to that of $G_{n}(s)$, i.e., $n_{r}$. However, this observation does not hold in time-domain DOB results. We can obtain from (10) that

$$
\begin{aligned}
& \operatorname{deg}\left(\operatorname{Hadj}(s I-S) K_{2}\right) \leq q-1 ; \\
& \operatorname{deg}\left(M_{n}(s)\right)=n-n_{r} ; \\
& \operatorname{deg}(\operatorname{det}(s I-(\tilde{A}-K \tilde{C})))=n+q,
\end{aligned}
$$

where $q$ and $n$ are the dimension of system matrix $S$ and $A$ of disturbance model (3) and system model (2). The minimum relative degree of $G_{u \hat{d}}(s)$ happens when $\operatorname{deg}(\operatorname{Hadj}(s I-$ $\left.S) K_{2}\right)=q-1$, and equals to

$$
n+q-\left(q-1+n-n_{r}\right)=n_{r}+1,
$$

which is larger than that of frequency-domain DOB by 1 .

\subsubsection{Disturbance types}

In addition, from the proof of (12) (see Appendix B), we can see that $G_{u \hat{d}}(0)=1$ holds only when

$$
D=B, \text { and } \operatorname{det}(S)=0,
$$

which means the frequency-domain DOB using a low-pass filter $Q(s)$ with unity dc gain in Fig. 1 can only effectively handle matched Type I disturbances rather than generic disturbance or mismatched disturbances.

So a new type of time-domain DOB (termed Functional Disturbance OBserver (FDOB)) is proposed to reduce the observer order by combing the idea of augmenting system states with disturbance states and functional observer theory in [18-20]. The frequency-domain DOBs can also be designed to handle more types of disturbances (mismatched disturbance and Type II disturbances) using FDOB techniques and its frequency-domain counterpart through transfer function realization.

\section{Functional Disturbance OBserver (FDOB)}

The basic philosophy of FDOB is that since part of system states are directly available by measurement $y=\tilde{C} \tilde{x}$, there is no need estimating that part of states and sometimes even part of the unmeasurable states do not need to be estimated for the purpose of disturbance estimation [23]. We only need estimate $L \tilde{x}$ rather than $\tilde{x}$, where $L$ is designed in a special structure

$$
L=\left[\begin{array}{cc}
L_{0} & O \\
O & I_{q}
\end{array}\right],
$$

where the gain matrix $L_{0}$ in $L$ lies in the orthogonal complement space of measurement matrix $C$ and so $\left[L^{T} \tilde{C}^{T}\right]^{T}$ has a full row-rank. When $L \tilde{x}$ is obtained, we can obtain the disturbance estimate $\hat{d}=\tilde{C} L \tilde{x}$ with $\tilde{C}=\left[O, I_{q}\right]$. In the following part, we will introduce the FDOB in terms of observer structure, transfer function realization, existence condition and design procedure respectively.

\subsection{Observer structure}

To develop a FDOB, we combine the idea of augmenting the system state with disturbance state and the functional observer theory in [20]. After choosing $L$ in the special form as in (19), the FDOB for linear system (4) has the form,

$$
\left\{\begin{array}{l}
\dot{z}=F z+G y+T u, \hat{\xi}=z+J y, \\
\hat{\omega}=\tilde{C} \hat{\xi}, \hat{d}=H \hat{\omega}
\end{array},\right.
$$

where the gain matrices are designed such that the following matrix identities hold:

$$
\left\{\begin{array}{l}
W \tilde{A}=F W+G \tilde{C}, W=L-J \tilde{C}, \\
T=W \tilde{B}, F \text { is stable }
\end{array},\right.
$$

Under condition (21), one can prove that the estimation error $e=L \tilde{x}-\hat{\xi}$ converges to zero and consequently $\hat{\xi}$ is the estimate of the state function $L \tilde{x}$ (e.g., [20]).

\subsection{Transfer function realisation}

Frequency-domain analysis is performed on FDOB (20) such that the relationship between FDOB and frequencydomain DOB can be investigated, upon which the FDOB in time-domain can be implemented in frequency-domain using the transfer functions. We can obtain the transfer functions $G_{u \hat{d}}(s)$ and $G_{y \hat{d}}(s)$ based on Laplace transformation of (20), given by

$$
\begin{gathered}
\hat{d}(s)=-G_{u \hat{d}}(s) u(s)+G_{y \hat{d}}(s) y(s), \\
G_{u \hat{d}}(s)=-H \tilde{C}[s I-F]^{-1} T \\
G_{y \hat{d}}(s)=H \tilde{C}\left[(s I-F)^{-1} G+J\right] .
\end{gathered}
$$

To explicitly find out the relationship between the transfer functions $G_{u \hat{d}}(s), G_{y \hat{d}}(s)$ and the system/disturbance model (2), (3), theoretical analysis is performed on (22) and (23) and the results are summarized in Theorem 3.

Theorem 3. For linear system (2) with disturbance model (3), if the DOB is designed using FDOB (20), then the transfer functions from input $u(s)$ and measurement $y(s)$ to disturbance estimate $\hat{d}(s)$ are given by

$$
\begin{aligned}
G_{u \hat{d}}(s) & =\frac{\operatorname{Hadj}(s I-S) J_{2} M_{n}(s)}{\operatorname{det}(s I-F)}, \\
G_{y \hat{d}}(s) & =\frac{\operatorname{Hadj}(s I-S) J_{2} N_{n}(s)}{\operatorname{det}(s I-F)} .
\end{aligned}
$$

The proof is given in Appendix E. Similar to Theorem 1, the disturbance model (i.e., the pair $(S, H)$ ) is also explicitly reflected in the transfer functions (24) and (25). 
The comparison analysis between FDOB and the frequency-domain DOB in Fig. 2 are performed in the following ways.

i. First, similar to the case of the time-domain DOB, the denominator of (24) can also be factored into $\operatorname{det}(s I-$ $F)=L_{1}(s) L_{2}(s)$, then (24) and (25) can be put into the same format as $M(s) K(s)$ and $N(s) K(s)$ in Fig. 2 by treating $K_{n}(s):=\operatorname{Hadj}(s I-S) J_{2}$;

ii. Second, the frequency-domain structure of FDOB is the same as that of the time-domain DOB in Fig. 3 and consequently the same as that of Fig. 2;

iii. Third, we can also prove that

$$
G_{u \hat{d}}(0)=1,
$$

under conditions $D=B$ and $\operatorname{det}(S)=0$. The proof is given in Appendix F.

iv. Finally, the following identity can be established

$$
\frac{1}{1-G_{u \hat{d}}}=\frac{\operatorname{det}(s I-F)}{\operatorname{det}\left(s I-A_{4}+J_{1} A_{4}\right) \operatorname{det}(s I-S)} .
$$

The proof is similar to that of (13) and so omitted here. We can see from (27) that $1 /\left(1-G_{u \hat{d}}\right)$ includes the generic disturbance model information $1 / \operatorname{det}(s I-$ $S$ ), which is consistent with the conclusion of [1] for the purpose of generic disturbance estimation through frequency-domain DOB.

Remark 4: Compared $G_{u \hat{d}}$ of the time-domain DOB (10) with that of FDOB (24), we can see that the numerators of them are the same, however, the denominator has been changed from $\operatorname{det}(s I-(\tilde{A}-K \tilde{C}))$ to $\operatorname{det}(s I-F)$. The dimension of $F$ equals to the row rank of $L=\left[\begin{array}{cc}L_{0} & O \\ O & I\end{array}\right]$ and $L_{0}$ has a full row-rank and so the relative degree of $G_{u \hat{d}}$ of the FDOB (24) is less than that of the time-domain DOB (10). More importantly, we can see that the observer order of FDOB, i.e., the row number of $\mathrm{L}$ is smaller than that of the traditional time-domain DOB, i.e., the order of $\tilde{A}$. Consequently, the proposed FDOB can reduce the DOB order, especially when multiple measurements are available. However, to reduce the observer order of FDOB, one can not choose an $L$ with an arbitrarily small row number. The $L$ should be selected such that the FDOB existence condition is satisfied, which is summarized below.

\subsection{Existence condition}

As we have transformed the disturbance observer design problem into the functional observer design, the existing results in [20] can be drawn to derive the existence condition of the proposed FDOB.

The first condition is on unnecessary state (the states that do not need to be estimated) decoupling, which is given by

$$
\operatorname{rank}\left(\left[\begin{array}{c}
L \tilde{A} \\
\tilde{C} \tilde{A} \\
\tilde{C} \\
L
\end{array}\right]\right)=\operatorname{rank}\left(\left[\begin{array}{c}
\tilde{C} \tilde{A} \\
\tilde{C} \\
L
\end{array}\right]\right),
$$

The second condition is on stability of $F$. Using geometric analysis, $F$ can be represented by

$$
F=U-Z V,
$$

$$
\begin{gathered}
U=L \tilde{A} L^{+}-L \hat{A} \Sigma^{+} \Psi \\
V=\left(I-\Sigma \Sigma^{+}\right) \Psi
\end{gathered}
$$

where $\hat{A}=\tilde{A}\left(I-L^{+} L\right), \Psi=\left[\begin{array}{c}\tilde{C} \tilde{A} L^{+} \\ \tilde{C} L^{+}\end{array}\right], \Sigma=\left[\begin{array}{c}\tilde{C} \hat{A} \\ \hat{C}\end{array}\right]$, and $Z$ being any compatible matrix.

From [20], one can obtain that $F$ being Hurwitz (or the pair $(\mathrm{U}, \mathrm{V})$ is detectable) is equivalent to

$$
\operatorname{rank}\left(\left[\begin{array}{c}
s L-L \tilde{A} \\
\tilde{C} \tilde{A} \\
\tilde{C}
\end{array}\right]\right)=\operatorname{rank}\left(\left[\begin{array}{c}
\tilde{C} \tilde{A} \\
\tilde{C} \\
L
\end{array}\right]\right), \forall s \in C, \operatorname{Re}(s) \geq 0 .
$$

To summarize, the existence conditions for the proposed FDOB are (28) and (32).

\subsection{Design procedure}

The design procedure of FDOB is based on the functional observer design procedure in [20], given by

i. Matrices $U$ and $V$ are firstly obtained from (30) and (31);

ii. $Z$ and $F$ can be obtained from (29) based on pole assignment of $U$ and $V$;

iii. The matrices $J$ and $K$ can be obtained from $[J, K]=$ $L \hat{A} \Sigma^{+}+Z\left(I-\Sigma \Sigma^{+}\right)$, and $G$ can be obtained from $G=$ $K+F J$

iv. Matrix $T$ can be obtained from $T=(L-J \tilde{C}) \tilde{B}$.

\section{Applications of the results}

Based on the frequency-domain analysis results (see, Theorem 1 and Theorem 3), we can extend the application scope (handling mismatched/Type II disturbances) of frequency-domain DOBs using the frequency-domain counterparts of FDOB, which is summarized below:

Step 1: Design DOBs in time-domain (e.g., FDOB), since they can handle matched, mismatched and generic disturbances;

Step 2: Calculate two transfer functions $G_{u \hat{d}}$ and $G_{y \hat{d}}$ based on the results of Theorem 1 and 3;

Step 3: Implement the DOBs using the diagram in Fig. 3 based on the obtained two transfer functions.

\section{Examples}

In this section, two sets of examples are given to illustrate the main findings of this paper. In the first example, a non-minimum phase system with different kinds of disturbances are considered including matched step disturbance, mismatched step disturbance and matched harmonic disturbance. This example is to illustrate the results in Sections 4 and 5. Then the proposed FDOB is then applied to the disturbance estimation and rejection control (termed FDOBC) problem for a rotary mechanical system of non-minimum phase.

\subsection{Numerical example}

Consider an unstable non-minimum phase system

$$
G(s)=\frac{5(s-9)}{s(s-25)} \text {. }
$$



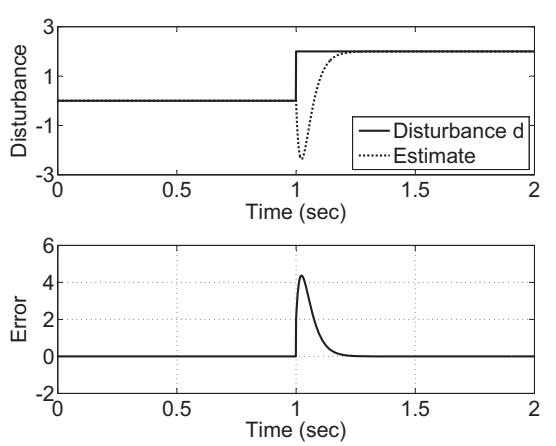

Fig. 4. Matched step disturbance estimation and its error.

Its sate-space model with disturbance $d$ is given by

$$
\left\{\begin{array}{l}
{\left[\begin{array}{l}
\dot{x}_{1} \\
\dot{x}_{2}
\end{array}\right]=\left[\begin{array}{ll}
0 & 1 \\
0 & 25
\end{array}\right]\left[\begin{array}{l}
x_{1} \\
x_{2}
\end{array}\right]+\left[\begin{array}{c}
5 \\
80
\end{array}\right] u+\left[\begin{array}{c}
5 \\
80
\end{array}\right] d} \\
y=\left[\begin{array}{ll}
1 & 0
\end{array}\right]
\end{array}\right.
$$

Suppose a state feedback controller $u=[20.67-2.37] x$ has been pre-designed with initial state $x_{1: 2}(0)=[0 ; 5]$.

\subsubsection{Matched disturbance}

We first consider the matched step disturbance based on the proposed FDOB, i.e., $S=0, H=1$ in disturbance model (3). The $L_{0}$ in functional matrix (19) is chosen as $L_{0}=\left[\begin{array}{ll}0 & 1\end{array}\right]$, based on which the conditions (28) and (32) are satisfied. Then following the design procedure in Section 4.4, we can calculate $U, V$. Choosing the poles of $F$ as $p_{1}=-30, p_{2}=$ -40 , we can calculate the corresponding $Z, F$ and then the rest of the matrices $J, G, T$. The initial states of the FDOB (20) are chosen as $[5 ; 0]$. Simulation result of the disturbance estimate is shown in Fig. 4.

We can see from Fig. 4 that the proposed FDOB can exponentially asymptotically estimate the step disturbance, while the disturbance estimate is zero in the absence of disturbance during the first $1 \mathrm{sec}$, i.e., normal control performance recovery $[2,4,7]$.

Besides, we can calculate the transfer functions from control input and output to disturbance estimate, given by

$$
G_{u \hat{d}}(s)=\frac{-400(s-9)}{3(s+30)(s+40)}, G_{y \hat{d}}(s)=\frac{-80(s-25)}{3(s+30)(s+40)} .
$$

We can verify that $G_{u \hat{d}}(s) / G_{y \hat{d}}(s)=G(s)$ and $G_{u \hat{d}}(0)=1$. Besides, the relative degree of $G_{u \hat{d}}(s)$ is 1 , which is equal to that of the original system (33). Furthermore, the observer order of FDOB equals to the dimension of original plant. That means there exists a disturbance observer in state-space, where the relative degree of $G_{u \hat{d}}$ and the order of the observer are equal to those of the frequency-domain DOB for this unstable, non-minimum phase system.

\subsubsection{Mismatched disturbance}

We then consider mismatched step disturbance. The disturbance distribution matrix is changed to $D=\left[\begin{array}{ll}0 & 80\end{array}\right]^{T}$ and the matrices $S, H, L_{0}$ are selected the same as that of Section 6.1.1. The simulation result is shown in Fig. 5

We can see from Fig. 5 that the proposed FDOB can exponentially asymptotically estimate the mismatched step disturbance. We can also calculate the corresponding trans-
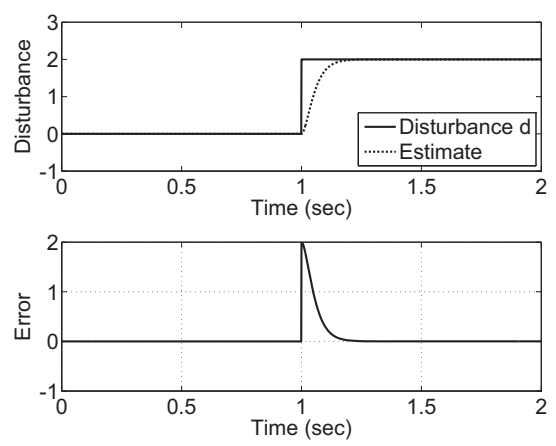

Fig. 5. Mismatched step disturbance estimation and its error.
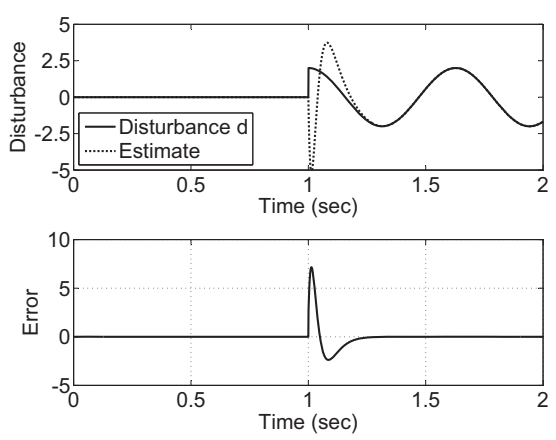

Fig. 6. Harmonic disturbance estimation and its error.

fer functions, given by

$$
G_{u \hat{d}}(s)=\frac{75(s-9)}{(s+30)(s+40)}, G_{y \hat{d}}(s)=\frac{15 s(s-25)}{(s+30)(s+40)} .
$$

One can verify that $G_{u \hat{d}}(s) / G_{y \hat{d}}(s)=G(s), \quad G_{u \hat{d}}(0)=$ $-675 / 1200 \neq 1$, which verifies the observation in Section 5.

\subsubsection{Harmonic disturbance}

The matched harmonic disturbance $d=2 \sin (10 t), t \geq$ $1 \mathrm{sec}$ is then considered. It can be put into the state space model (3) with $S=\left[\begin{array}{cc}0 & 10 \\ -10 & 0\end{array}\right], H=\left[\begin{array}{ll}1 & 0\end{array}\right]$ and initial value $\omega_{0}=\left[\begin{array}{ll}2 & 0\end{array}\right]$.

The matrix $L_{0}$ is chosen as $L_{0}=\left[\begin{array}{ll}0 & 1\end{array}\right]$. Choosing the poles of $F$ as $p_{1}=-30, p_{2}=-40, p_{3}=-50$ and the rest of the design procedure is the same as that of Section 6.1.1, the simulation result is shown in Fig. 6

We can see from Fig. 6 that the proposed FDOB can exponentially asymptotically estimate the harmonic disturbance with unknown amplitude and phase. The transfer functions $G_{u \hat{d}}(s)$ and $G_{y \hat{d}}(s)$ can be calculated. We can verify that $G_{u \hat{d}}(s) / G_{y \hat{d}}(s)=G(s)$. However $G_{u \hat{d}}(0)=-0.0232 \neq$ 1 , which verifies the observation in Section 5. For the case of Type II disturbance, the traditional frequency-domain design method for DOBs can not be applied, but we can implement the FDOB in frequency-domain using the procedure given in Section 5 .

\subsection{Practical example}

In this section, the proposed FDOB is applied to the disturbance estimation and rejection control (termed FDOBC) 

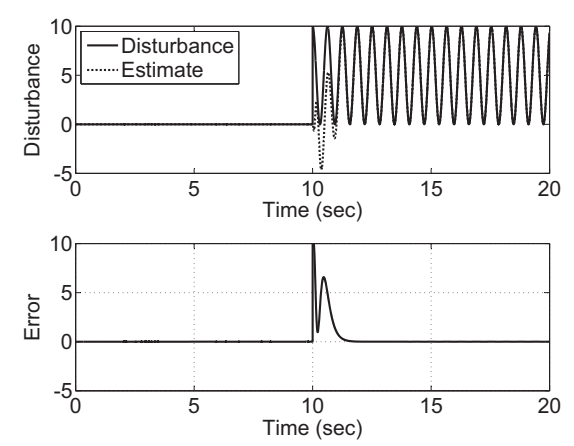

Fig. 7. Biased harmonic disturbance estimation using FDOB.
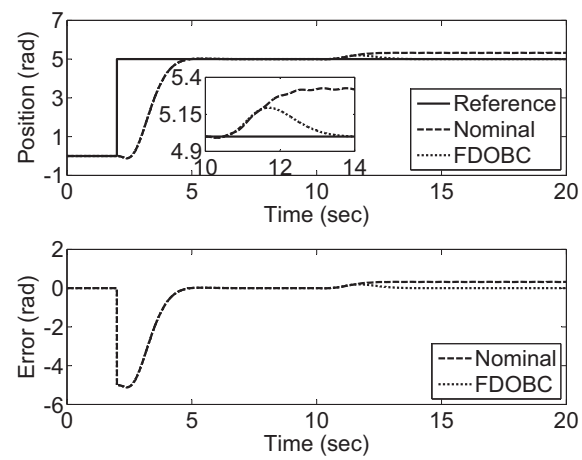

Fig. 8. Position control with and without disturbance compensation.

problem for a rotary mechanical system of non-minimum phase from [27]. The system is given by

$$
G(s)=\frac{1.202(4-s)}{s(s+9)\left(s^{2}+12 s+56.25\right)} .
$$

A normal controller has been pre-designed in state space such that the closed-loop poles are $-3,-2 \pm 1.5 i,-4$. A step reference position with amplitude 5 is supposed to act on the system at $2 \mathrm{sec}$. At $10 \mathrm{sec}$, a harmonic disturbance with unknown amplitude, phase and bias is supposed to act on the system, which is given by $d=5+5 \sin (10 t)$.

The proposed FDOB is used to estimate the disturbance, where the poles of matrix $F$ is selected as $-5,-10,-15$, $-20,-25,-30$. We further reject its effect by direct feedforward the disturbance estimate based on the principle of DOBC (see, $[2,7,8])$. The disturbance estimation performance is shown in Fig. 7. The position control results with and without disturbance compensation (i.e., under FDOBC and nominal control) are given in Fig. 8.

We can see from Fig. 7 that the proposed FDOB can asymptotically estimate the harmonic disturbance with unknown amplitude, phase and bias. We can see from Fig. 8 that the FDOBC can effectively remove the effect of unknown disturbances (with preservation of the nominal control performance), while the nominal controller results in control error.

\section{Conclusions}

This paper provides a generic analysis of the relationship between time/frequency-domain DOBs. The traditional frequency-domain DOB structure using a low pass filter with unity gain can only effectively handle disturbances satisfying matching condition, while the time-domain DOB generates a higher order observer. A Functional Disturbance OBserver (FDOB) is proposed to improve the time-domain DOB. Compared with the frequency-domain DOBs, the proposed FDOB can handle more types of disturbances, while compared with the existing time-domain DOBs the proposed FDOB generates an observer with lower order. Numerical examples including a rotary mechanical system of nonminimum phase are presented to verify the findings. The proposed FDOB has potentials in the fields of both disturbance rejection control and fault diagnosis [28].

\section{Acknowledgements}

This work was jointly funded by UK Engineering and Physical Sciences Research Council (EPSRC) and BAE Systems (EP/H501401/1). The third author was supported by National Natural Science Foundation of China under Grant 61573099, Natural Science Foundation of Jiangsu Province under Grants BK2012327, and Fundamental Research Funds for the Central Universities.

\section{Appendix A: Proof of Theorem 1}

We first consider transfer function $G_{u \hat{d}}$. From the identity $A^{-1}=\operatorname{adj}(A) / \operatorname{det}(A)$, one can obtain:

$G_{u \hat{d}}=-H \tilde{C}[s I-(\tilde{A}-K \tilde{C})]^{-1} \tilde{B}=\frac{-[O, H] a d j(s I-(\tilde{A}-K \tilde{C})) \tilde{B}}{\operatorname{det}(s I-(\tilde{A}-K \tilde{C}))}$.

In addition, for SISO system $(A, B, C)$, the following property holds,

$$
\operatorname{Cadj}(s I-A) B=\left|\begin{array}{cc}
s I-A & B \\
-C & 0
\end{array}\right| .
$$

Partitioning the gain matrix $K$ into $K=\left[K_{1}^{T}, K_{2}^{T}\right]^{T}$ in conjunction with (35), the numerator of (34) is

$$
\begin{aligned}
& -[O, H] \operatorname{adj}[s I-(\tilde{A}-K \tilde{C})] \tilde{B} \\
& =-\left|\begin{array}{ccc}
s I-A+K_{1} C-D H & B \\
K_{2} C & s I-S & O \\
O & -H & 0
\end{array}\right| \\
& =-\left|\begin{array}{ccc}
O & -D H & -K_{1} \\
O & s I-S-K_{2} \\
O & -H & 0
\end{array}\right|\left|\begin{array}{ccc}
s I-A & O & B \\
O & I & O \\
-C & O & 0
\end{array}\right| \\
& =H a d j(s I-S) K_{2} C a d j(s I-A) B .
\end{aligned}
$$

We then consider transfer function $G_{y \hat{d}}$. Similar to (34) and (36), one can obtain the following two identities,

$$
\begin{aligned}
G_{y \hat{d}}=H \tilde{C}[ & s I-(\tilde{A}-K \tilde{C})]^{-1} K=\frac{[O, H] \operatorname{adj}[s I-(\tilde{A}-K \tilde{C})] K}{\operatorname{det}(s I-(\tilde{A}-K \tilde{C}))} . \\
& =\left|\begin{array}{ccc}
s I-A] \operatorname{adj}[s I-(\tilde{A}-K \tilde{C})] K \\
K_{2} C & s I-S & K_{2} \\
O & -H & 0
\end{array}\right| \\
& =-\left|\begin{array}{ccc}
I-D H & -K_{1} \\
O & s I-S-K_{2} \\
O & -H & 0
\end{array}\right|\left|\begin{array}{ccc}
s I-A & O & O \\
O & I & O \\
-C & O & 1
\end{array}\right| \\
& =H a d j(s I-S) K_{2} \operatorname{det}(s I-A) .
\end{aligned}
$$


Substituting (36) into (34), and (38) into (37) ends the proof.

\section{Appendix B: Proof of (12)}

To prove $G_{u \hat{d}}(0)=1$, we only need to prove the subtraction of the denominator and numerator of (34) is zero at $s=0$, which is given as follows:

$$
\begin{aligned}
& \left|\begin{array}{ccc}
-A+K_{1} C & -D H & B \\
K_{2} C & -S & O \\
O & -H & 0
\end{array}\right|+\left|\begin{array}{cc}
-A+K_{1} C & -D H \\
K_{2} C & -S
\end{array}\right| \\
& =\left|\begin{array}{ccc}
-A+K_{1} C & -D H & B \\
K_{2} C & -S & O \\
O & -H & 0 \\
-A+K_{1} C & -D H & B \\
K_{2} C & -S & O \\
O & -H & 1
\end{array}\right|+\left|\begin{array}{ccc}
-A+K_{1} C & -D H & O \\
K_{2} C & -S & O \\
O & -H & 1 \\
-A+K_{1} C & O & D \\
K_{2} C & -S & O \\
O & O & 1
\end{array}\right| \\
& =\operatorname{det}\left(-A+K_{1} C\right) \operatorname{det}(-S) \text {, }
\end{aligned}
$$

where the identity $D=B$ has been used in the third equality.

The proof ends since $\operatorname{det}(S)=0$.

\section{Appendix C: Proof of (13)}

From (34), one can obtain the denominator of $1 /(1-$ $\left.G_{u \hat{d}}\right)$, given by

$$
\begin{aligned}
& \operatorname{det}(s I-(\tilde{A}-K \tilde{C}))+[O, H] \operatorname{adj}(s I-(\tilde{A}-K \tilde{C})) \tilde{B} \\
& \begin{array}{l}
=\left|\begin{array}{ccc}
s I-A+K_{1} C & -D H & O \\
K_{2} C & s I-S & O \\
O & -H & 1 \\
s I-A+K_{1} C & -D H & B \\
K_{2} C & s I-S & O \\
O & -H & 1
\end{array}\right|+\left|\begin{array}{ccc}
s I-A+K_{1} C & -D H & B \\
K_{2} C & s I-S & O \\
O & -H & 0 \\
s I-A+K_{1} C & O & B \\
K_{2} C & s I-S & O \\
O & O & 1
\end{array}\right| \\
=\operatorname{det}\left(s I-A+K_{1} C\right) \operatorname{det}(s I-S),
\end{array}
\end{aligned}
$$

where in the third equality $D=B$ has been used.

\section{Appendix D: Proof of Theorem 2}

Using the well-known block matrix inversion, the following equation is derived

$$
\begin{aligned}
& \tilde{C}(s I-\tilde{A})^{-1} \\
& =[0 \mid 1]\left[\begin{array}{l|l}
s I-\left(A-L_{x} C\right) & -D \\
\hline L_{d} L_{x} C & s
\end{array}\right]^{-1} \\
& =[0 \mid 1]\left[\begin{array}{c|c}
* & * \\
\hline-\tilde{G}^{-1} L_{d} L_{x} C\left[s I-\left(A-L_{x} C\right)\right]^{-1} & \tilde{G}^{-1}
\end{array}\right] \\
& =\left[-\tilde{G}^{-1} L_{d} L_{x} C\left[s I-\left(A L_{x} C\right)\right]^{-1} \mid \tilde{G}^{-1}\right],
\end{aligned}
$$

where

$$
\tilde{G}=s+L_{d} L_{x} C\left[s I-\left(A-L_{x} C\right)\right]^{-1} D
$$

Combining Eqs. (15), (16) with (39) yields

$$
\begin{aligned}
G_{u \hat{d}}(s)= & -\tilde{G}^{-1} L_{d} L_{x} C\left[s I-\left(A-L_{x} C\right)\right]^{-1} B, \\
G_{y \hat{d}}(s)=- & \tilde{G}^{-1} L_{d} L_{x} C\left[s I-\left(A-L_{x} C\right)\right]^{-1} L_{x} \\
& +\tilde{G}^{-1} L_{d} L_{x} .
\end{aligned}
$$

\section{Proof of fact 1)}

Since the observer gains $L_{x}$ and $L_{d}$ can be chosen such that $\tilde{A}$ is Hurwitz matrix, it follows from (15) that transfer function $G_{u \hat{d}}(s)$ is stable.
It can be derived from (15) and (17) that

$$
Q(s)=-G_{u \hat{d}}(s),
$$

thus $Q(s)$ is also a stable transfer function.

Combining (41) with (43), yields

$$
Q(s)=\tilde{G}^{-1} L_{d} L_{x} C\left[s I-\left(A-L_{x} C\right)\right]^{-1} B,
$$

Under the condition $B=D$, combining (40) with (44), yields

$$
Q(0)=1 \text {. }
$$

It can be concluded that $Q(s)$ in (17) is a stable low-pass filter with unity dc gain as long as the observer gains are appropriately selected.

\section{Proof of fact 2)}

The transfer function of system (2) is

$$
P_{n}(s)=C(s I-A)^{-1} B .
$$

Combining (42) with (45), gives

$$
\begin{aligned}
& G_{y \hat{d}}(s) P_{n}(s) \\
&=\left\{-\tilde{G}^{-1} L_{d} L_{x} C\left[s I-\left(A-L_{x} C\right)\right]^{-1} L_{x}+\tilde{G}^{-1} L_{d} L_{x}\right\} \\
& \quad \times C(s I-A)^{-1} B \\
&=-\tilde{G}^{-1} L_{d} L_{x} C\left\{\left[s I-\left(A-L_{x} C\right)\right]^{-1} L_{x} C(s I-A)^{-1}\right. \\
&\left.-(s I-A)^{-1}\right\} B \\
&= \tilde{G}^{-1} L_{d} L_{x} C\left[s I-\left(A-L_{x} C\right)\right]^{-1} B \\
&=-G_{u \hat{d}}(s) .
\end{aligned}
$$

Considering (43) and (46), the required result can be easily obtained.

\section{Appendix E: Proof of Theorem 3}

Without loss of generality, we suppose $C=\left[1, O_{1 \times n-1}\right]$ (this assumption can always be satisfied for SISO system (2) through some non-singular linear transformation). Partitioning $\left[\begin{array}{ll}J & K\end{array}\right]$ into $\left[\begin{array}{ll}J_{1} & K_{1} \\ J_{2} & K_{2}\end{array}\right]$ and taking the specific structure of $L$ into account, the matrix $F$ can be put into the following form:

$$
\begin{aligned}
F= & L \tilde{A} L^{+}-[J, K]\left[\begin{array}{c}
\tilde{C} \tilde{A} L^{+} \\
\tilde{C} L^{+}
\end{array}\right] \\
= & {\left[\begin{array}{cc}
L_{0} & O \\
O & I
\end{array}\right]\left[\begin{array}{cc}
A & D H \\
O & S
\end{array}\right]\left[\begin{array}{cc}
L_{0}^{+} & O \\
O & I
\end{array}\right] } \\
& -\left[\begin{array}{ll}
J_{1} & K_{1} \\
J_{2} & K_{2}
\end{array}\right]\left[\begin{array}{cc}
C A L_{0}^{+} & C D H \\
C L_{0}^{+} & O
\end{array}\right] \\
= & {\left[\begin{array}{cc}
L_{0} A L_{0}^{+}-J_{1} C A L_{0}^{+}-K_{1} C L_{0}^{+} & L_{0} D H-J_{1} C D H \\
-J_{2} C A L_{0}^{+}-K_{2} C L_{0}^{+} & S-J_{2} C D H
\end{array}\right] . }
\end{aligned}
$$

Noticing that $L_{0}=[O, I]$ has a full-row rank and so $L_{0}^{+}=L_{0}^{T}$ and matrix $A, B$ can be partitioned into $\left[\begin{array}{ll}A_{1} & A_{1} \\ A_{3} & A_{4}\end{array}\right]$ and $\left[\begin{array}{l}B_{1} \\ B_{2}\end{array}\right]$, respectively, we can obtain the following matrix equalities:

$$
\begin{aligned}
& L_{0} A L_{0}^{+}=A_{4}, C A L_{0}^{+}=A_{2}, \\
& C L_{0}^{+}=0, L_{0} B=B_{2}, C B=B_{1}
\end{aligned}
$$


We first consider $G_{u \hat{d}}$. Similar to the proof of Theorem 1 , we can obtain the following identity.

$$
G_{u \hat{d}}=-H \tilde{C}(s I-F)^{-1} T=\frac{-H \tilde{C} a d j(s I-F) T}{\operatorname{det}(s I-F)} .
$$

Based on (48) in conjunction with the definition of $F$ in (47), the numerator of (49) is governed by

$$
\begin{aligned}
& H \tilde{C} a d j(s I-F) T \\
& =-\left|\begin{array}{ccc}
s I-A_{4}+J_{1} A_{2} & -L_{0} D H+J_{1} C D H & B_{2}-J_{1} B_{1} \\
J_{2} A_{2} & s I-S+J_{2} C D H & -J_{2} B_{1} \\
O & -H & O
\end{array}\right| \\
& =-\mid \begin{array}{ccc}
s I-A_{4}+J_{1} A_{2} & O & B_{2}-J_{1} B_{1} \\
J_{2} A_{2} & s I-S & -J_{2} B_{1} \\
O & -H & O
\end{array} \\
& =-\left|\begin{array}{ccc}
I & -J_{1} & O \\
O & -J_{2} & -(s I-S) \\
O & O & H
\end{array}\right|\left|\begin{array}{ccc}
s I-A_{4} & O & B_{2} \\
-A_{2} & O & B_{1} \\
O & -I & O
\end{array}\right| \\
& =-\left|\begin{array}{cc}
-J_{2} & -(s I-S) \\
O & H
\end{array}\right|\left|\begin{array}{ccc}
O & -A_{2} & B_{1} \\
O & s I-A_{4} & B_{2} \\
-I & O & O
\end{array}\right| \\
& =(-1)^{2 q}\left|\begin{array}{cc}
(s I-S) & J_{2} \\
-H & O
\end{array}\right|\left|\begin{array}{ccc}
s I-A_{1} & -A_{2} & B_{1} \\
-A_{3} & s I-A_{4} & B_{2} \\
-I & O & O
\end{array}\right| \\
& =\operatorname{Hadj}(s I-S) J_{2} \operatorname{Cadj}(s I-A) B \text {. }
\end{aligned}
$$

Secondly, we consider $G_{y \hat{d}}$. Similar to the proof of Theorem 1 , we can obtain the following identity,

$$
\begin{aligned}
& G_{y \hat{d}}=H \tilde{C}\left[(s I-F)^{-1} G+J\right] \\
& =\frac{H \tilde{C} a d j(s I-F) G}{\operatorname{det}(s I-F)}+\frac{H \tilde{C} J \operatorname{det}(s I-F)}{\operatorname{det}(s I-F)},
\end{aligned}
$$

the numerator of which is as follows:

$$
\begin{aligned}
& H \tilde{C} \text { adj }(s I-F) G+H \tilde{C} J \operatorname{det}(s I-F) \\
& =\left|\begin{array}{cc}
s I-F & G \\
-H \tilde{C} & O
\end{array}\right|+\left|\begin{array}{cc}
s I-F & O \\
-H \tilde{C} & H \tilde{C} J
\end{array}\right|=\left|\begin{array}{cc}
s I-F & G \\
-H \tilde{C} & H \tilde{C} J
\end{array}\right| \\
& =\left|\begin{array}{ccc}
s I-F & s J-F J+G \\
-H \tilde{C} & O & |=| \begin{array}{cc}
s I-F & s J+K \\
-H \tilde{C} & O
\end{array} \mid \\
s I-A_{4}+J_{1} A_{2} & -L_{0} D H+J_{1} C D H & s J_{1}+K_{1} \\
J_{2} A_{2} & s I-S+J_{2} C D H & s J_{2}+K_{2} \\
O & -H & O \\
O & & -H \\
s I-A_{4}+J_{1} A_{2} & O & s J_{1}+K_{1} \\
J_{2} A_{2} & s I-S & s J_{2}+K_{2} \\
O & -H & O
\end{array}\right| .
\end{aligned}
$$

At this stage, we suppose the following identities hold (its proof will be given later):

$$
\left\{\begin{array}{l}
K_{1}=-J_{1} A_{1}+A_{3} \\
K_{2}=-J_{2} A_{1} .
\end{array}\right.
$$

Substituting (53) into (52) gives

$$
\begin{aligned}
& H \tilde{C} a d j(s I-F) G+H \tilde{C} J \operatorname{det}(s I-F)
\end{aligned}
$$

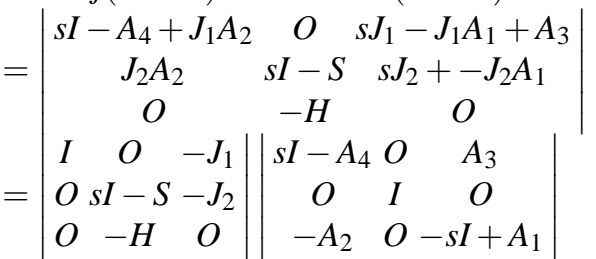

$$
\begin{aligned}
& =\operatorname{Hadj}(s I-S) J_{2} \operatorname{det}(s I-A) \text {. }
\end{aligned}
$$

Substituting (50) into (49), and (54) into (51) ends the proof.
Then we give the proof of (53), which is based on the Sylvester equation in (21). From the Sylvester equation, one can obtain

$$
G \tilde{C}=W \tilde{A}-F W
$$

Taking the structure of $\tilde{C}$ and $W$ into consideration, (55) is equivalent to

$$
\begin{aligned}
& {\left[\begin{array}{ccc}
G_{1} & O & O \\
G_{2} & O & O
\end{array}\right]=\left[\begin{array}{cc}
\left(L_{0}-J_{1} C\right) A & \left(L_{0}-J_{1} C\right) D H \\
-J_{2} C A & S-J_{2} C D H
\end{array}\right]} \\
& -\left[\begin{array}{cc}
A_{4}-J_{1} A_{2} & \left(L_{0}-J_{1} C\right) D H \\
-J_{2} A_{2} & S-J_{2} C D H
\end{array}\right]\left[\begin{array}{ccc}
L_{0}-J_{1} C & O \\
-J_{2} C & I
\end{array}\right],
\end{aligned}
$$

based on which in conjunction with $\left[L_{0}-J_{1} C\right]=\left[-J_{1}, I\right]$, we can obtain

$$
\left\{\begin{aligned}
G_{1}= & A_{3}-A_{1} J_{1}+\left(A_{4}-J_{1} A_{2}\right) J_{1} \\
& +\left(L_{0} D H-J_{1} C D H\right) J_{2}, \\
G_{2}= & -J_{2} A_{1}-J_{2} A_{2} J_{1}+\left(S-J_{2} C D H\right) J_{2}
\end{aligned}\right.
$$

From $K=G-F G$, one can obtain

$$
\left\{\begin{array}{l}
K_{1}=G_{1}-F_{1} J_{1}-F_{2} J_{2}=A_{3}-A_{1} J_{1} \\
K_{2}=G_{2}-F_{3} J_{1}-F_{4} J_{2}=-J_{2} A_{1}
\end{array}\right.
$$

This ends the proof.

\section{Appendix F: Proof of Eq. (26)}

To prove $G_{u \hat{d}}(0)=1$, we only need to prove the subtraction of the numerator and denominator of (49) is zero at $s=0$, which is given as follows:

From (50), the denominator minus the numerator is

$$
\begin{aligned}
& \left|\begin{array}{ccc}
-A_{4}+J_{1} A_{2} & O & B_{2}-J_{1} B_{1} \\
J_{2} A_{2} & -S & -J_{2} B_{1} \\
O & -H & O
\end{array}\right| \\
& +\left|\begin{array}{ccc}
-A_{4}+J_{1} A_{2} & -L_{0} D H+J_{1} C D H & O \\
J_{2} A_{2} & -S+J_{2} C D H & O \\
O & -H & I
\end{array}\right| \\
& =\left|\begin{array}{ccc}
-A_{4}+J_{1} A_{2} & O & B_{2}-J_{1} B_{1} \\
J_{2} A_{2} & -S & -J_{2} B_{1} \\
O & -H & O \\
-A_{4}+J_{1} A_{2} & O & -L_{0} D+J_{1} C D \\
J_{2} A_{2} & -S & J_{2} C D \\
O & -H & I
\end{array}\right| .
\end{aligned}
$$

When $D=B$ and consequently $J_{2} C D=J_{2} B_{1}$ and $-L_{0} D+J_{1} C D=-B_{2}+J_{1} B_{1}$, the above equation can be further calculated as

$$
\begin{aligned}
& \left|\begin{array}{ccc}
-A_{4}+J_{1} A_{2} & O & O \\
J_{2} A_{2} & -S & O \\
O & -H & I
\end{array}\right|=\left|\begin{array}{cc}
-A_{4}+J_{1} A_{2} & O \\
J_{2} A_{2} & -S
\end{array}\right| \\
& =\operatorname{det}\left(-A_{4}+J_{1} A_{2}\right) \operatorname{det}(-S) .
\end{aligned}
$$

The proof ends since $\operatorname{det}(S)=0$.

\section{References}

[1] Schrijver, E., and Van Dijk, J., 2002, "Disturbance Observers for Rigid Mechanical Systems: Equivalence, Stability, and Design,” ASME J. Dyn. Syst., Meas., Control, 124(4), pp. 539548.

[2] Li, S., Yang, J., Chen, W. H., and Chen, X., 2014, "Disturbance Observer-based Control: Methods and Applications," CRC Press, Boca Raton, USA.

[3] Guo, L., and Cao, S., 2013, "Anti-disturbance Control for 
Systems With Multiple Disturbances," CRC Press, Boca Raton, USA.

[4] Chen, W. H., Yang, J., Guo, L. and Li, S., 2016, "Disturbance observer-based control and related methods: An overview,” IEEE Trans. Ind. Electron., 63(2), pp. 1083-1095.

[5] Su, J., Li, B., Chen, W. H., 2015, “On Existence, Optimality and Asymptotic Stability of the Kalman Filter With Partially Observed Inputs," Automatica, 53, pp. 149-154.

[6] Chen, W. H., Yang, J., Zhao, Z, 2016, "Robust Control of Uncertain Nonlinear Systems: A Nonlinear DOBC Approach, " ASME J. Dyn. Syst., Meas., Control, accepted.

[7] Chen, W. H., Ballance, D., Gawthrop, P., and O'Reilly, J., 2000, "A Nonlinear Disturbance Observer for Robotic Manipulators," IEEE Trans. Ind. Electron., 47(4), pp. 932-938.

[8] Yang, J., Li, S., Su, J., and Yu, X., 2013, "Continuous Nonsingular Terminal Sliding Mode Control for Systems with Mismatched Disturbances," Automatica, 49(7), pp. 2287-2291.

[9] Johnson, C., 1968, "Optimal Control of the Linear Regulator with Constant Disturbances," IEEE Trans. Automa. Control, 13(4), pp. 416-421.

[10] Han, J., 2009, "From PID to Active Disturbance Rejection Control," IEEE Trans. Ind. Electron., 56(3), pp. 900-906.

[11] Su, J., Li, B., and Chen, W. H., 2015, "Simultaneous State and Input Estimation With Partial Information on the Inputs," Systems Science \& Control Engineering, 3(1), pp. 445-452.

[12] Ohishi, K., Nakao, M., Ohnishi, K., and Miyachi, K., 1987, "Microprocessor-controlled DC Motor for Load-Insensitive Position Servo System,” IEEE Trans. Ind. Electron., 34(1), pp. 4449.

[13] Shim, H., and Jo, N., 2009, "An Almost Necessary and Sufficient Condition for Robust Stability of Closed-loop Systems with Disturbance Observer," Automatica, 45(1), pp. 296-299.

[14] Park, G., Joo, Y., and Shim, H., 2014, “Asymptotic rejection of sinusoidal disturbances with recovered nominal transient performance for uncertain linear systems," IEEE Conference on Decision and Control, pp. 4404-4409.

[15] Joo, Y., Park, G., Back, J., and Shim, H., 2016, "Embedding internal model in disturbance observer with robust stability," IEEE Trans. Automa. Control, available online.

[16] Su, J., Yang, J., and Li, S, 2013, "Continuous finite-time anti-disturbance control for a class of uncertain nonlinear system," Trans. Inst. Meas. Control, 36(3), pp. 300-311.

[17] Xiong, Y., and Saif, M. 2003), "Unknown Disturbance Inputs Estimation Based on A State Functional Observer Design," Automatica, 39(8), pp. 1389-1398.

[18] Kim, K., and Rew, K., 2013, "Reduced Order Disturbance Observer for Discrete-Time Linear System," Automatica, 49(4), pp. 968-975.

[19] Su, J., Li, B., Chen, W. H., and Yang, J., 2016, "ReducedOrder Disturbance Observer Design for Discrete-Time Linear Stochastic Systems," Trans. Inst. Meas. Control, available online.

[20] Darouach, M., 2000, "Existence and Design of Functional Observers for Linear Systems," IEEE Trans. Automa. Control, 45(5), pp. 940-943.

[21] Yang, J., Su, J., Li, S., and Yu, X., 2014, "Highorder Mismatched Disturbance Compensation for Motion Control Systems Via a Continuous Dynamic Sliding-mode Approach,” IEEE Trans. Ind. Inform., 10(1), 604-614.

[22] Chen, W. H., 2004, "Disturbance Observer Based Control for Nonlinear Systems," IEEE/ASME Trans. Mechatron., 9(4), pp. 706-710.

[23] Su, J., Chen, W. H, and Li, B., 2015, "High Order Disturbance
Observer Design for Linear and Nonlinear Systems," Proc. Int Conf. on Information and Automation, pp. 1893-1898.

[24] Miklosovic, R., Radke, A., and Gao, Z. 2006, "Discrete Implementation and Generalization of the Extended State Observer," Proc. of American Control Conf, pp. 2209-2214.

[25] Jiang, G., Wang, S.,and Song, W., 2000, "Design of Observer with Integrators for Linear Systems with Unknown Input Disturbances," Electron. Lett., 36(13), pp. 1168-1169.

[26] Huang, Y., and Xue, W., 2014, "Active Disturbance Rejection Control: Methodology and Theoretical Analysis," ISA transactions, 53(4), pp. 963-976.

[27] Wang, L., Freeman, C., Rogers, E., and Owens, D., 2010, "Experimentally Validated Continuous-Time Repetitive Control of Non-minimum Phase Plants with A Prescribed Degree of Stability," Control Eng. Pract., 18(10), pp. 1158-1165.

[28] Su, J., and Chen, W. H., 2016, "Fault diagnosis for vehicle lateral dynamics with robust threshold," Proc. of IEEE International Conference on Industrial Technology, available online. 